eigenen Nachwuchspreis zu verzichten und diese Initiative der KSR zu überlassen. Prof. Weisensee bekräftigt diese Entscheidung in seinem Schreiben vom 12. April 2013: „Die DGfK ist stolz und glücklich darüber, dass es den Ravenstein-Förderpreis zur Unterstützung des kartographischen Nachwuchses gibt und steht zu ihrem „Selbstbindungsbeschluss“. Das funktioniert seit über 16 Jahren reibungslos und soll - so meine Empfehlung - auch künftig so bleiben.

Denn (ich sage das nicht nur in eigener Sache): ich suche (m)einen Nachfolger/Nachfolgerin. Seit über 50 Jahren Mitglied, davon 35 Jahre der DGfK ehrenamtlich verbunden - als Ausrichter des 31. DKT 1982, 12 Jahre als Schatzmeister und für sechs Jahre Kassenprüfer sowie seit 25 Jahren zunächst als Verwalter und dann als Vorsitzender des Vorstands der Stiftung für den „Ravenstein-Förderpreis“ - werde ich zum 1. Juli 2017 diese Aufgabe (dann schon 75 Jahre alt) abgeben.

Das Profil meines Nachfolgers sollte sein: Entweder Kartograph oder aus einem verwandten Beruf der Geowissenschaften mit Interesse an administrativ-rechtlichen Aufgaben. Dazu gehören Kontakte und Berichte an die Stiftungsaufsicht, an die Finanzbehörden, die Organisation der alljährlichen Preisvergabe, die Einladungen und Abstimmungen hierzu, die Information der Preisträger (gemeinsam mit dem JuryVorsitzenden), die Erhaltung des Vermögens und der Abgleich aller finanziellen Grundlagen mit dem Vorstand der „Helga Ravenstein-Stiftung“. Ich hoffe und wünsche, dass sich hierfür eine Persönlichkeit findet, die ich gerne berate.

Und so lege ich mit diesem Beitrag quasi einen Leistungsbericht vor, der neben der Information zur Entstehung des Förderpreises und in Erinnerung an eine großartige Frau, Helga Ravenstein, auch Rechenschaft über mein Tun für ein Vierteljahrhundert
Stiftungsarbeit ablegt. Er ist zugleich ein Beweis für Teamarbeit und das gemeinsame Ziel: dem kartographischen Nachwuchs im weiteren Sinne eine hervorragende Möglichkeit der Präsentation seiner Leistungen zu geben und sich bundesweit zu vergleichen. Der hohe Stellenwert des Preises zeigte sich auch bei den Vorträgen zu Ausbildungsthemen anlässlich des Kartographentages 2016 in Potsdam - die Ausbildungsbetriebe nannten voller Stolz die Häufigkeit der Preisträger unter ihren Auszubildenden. Eines kann ich versichern: Die „Kartographie-Stiftung Ravenstein“ zu führen, die auf einem wirtschaftlich und rechtlich soliden Fundament steht, macht Freude. Und sie erhält jung, sind doch die Ansprechpartner um die 20.

Wer will also trotz respektablem Alter länger jung bleiben?

Er möge sich bitte melden!

Horst Schöttler

\section{Werner Herzog} im Ruhestand

Zwei Jahre, nachdem Werner Herzog nach 16 Jahren die Leitung der DGfK-Sektion RheinRuhr aus der Hand gegeben hat, folgt mit dem Ende des Sommersemesters 2016 nun auch sein beruflicher Abschied vom Geographischen Institut der RuhrUniversität Bochum.

Werner Herzog hat in Bochum Geographie mit den Nebenfächern Geologie, Sozial- und Wirtschaftsgeschichte sowie Kartographie/Vermessung und Volkswirtschaft studiert und nach dem Diplom mit einer empirischen Arbeit zur kartographischen Kommunikation bei Heinz Pape 1985 promoviert. Anschließend arbeitete er zunächst bei Heinz Pape und später bei dessen Nachfolgern Jean-Claude Müller und Frank Dickmann über dreiBig Jahre als Akademischer Rat bzw. Oberrat in der Arbeitsgruppe (AG) Geomatik des Geographi- schen Instituts. So wie sich heute die AG Geomatik (Kartographie) in der Forschung mit Problemen der Kartennutzung bzw. -effizienz beschäftigt, so steht Werner Herzog auch mit seinem fachlichen Engagement, z. B. in der DGfK-Kommission „Kartennutzung“, für die Kontinuität dieses Arbeitsfeldes an der Ruhr-Universität. In der Lehre hat Werner Herzog vor allem die Gebiete der amtlichen Geobasis- und -fachdaten, der Produktionsverfahren und der handlungsorientierten Gestaltung kartographischer Medien abgedeckt. Gerade diese sehr praxisorientierten Lehrveranstaltungen, zu denen Werner Herzog zahlreiche Exkursionen anbot, trafen immer auf reges Interesse bei den Studierenden. - Die Mitglieder der AG Geomatik und der Sektion Rhein-Ruhr wünschen einen geruhsamen Ruhestand. Glück auf!

Frank Dickmann

\section{Geburtstag} Heinz Kühr

Die ersten Absolventen der Fachrichtung Kartographie an der ehemaligen Ingenieurschule für Geodäsie und Kartographie in Dresden, die dort im Jahre 1957 ihre Examen bestanden hatten, gratulierten und ehrten ihren lebensältesten Kommilitonen und väterlichen Nestor Heinz Kühr zu seinem 90. Geburtstag am 26.4.2016 in Dresden. Zur Feier in das Restaurant „Körner Gar-

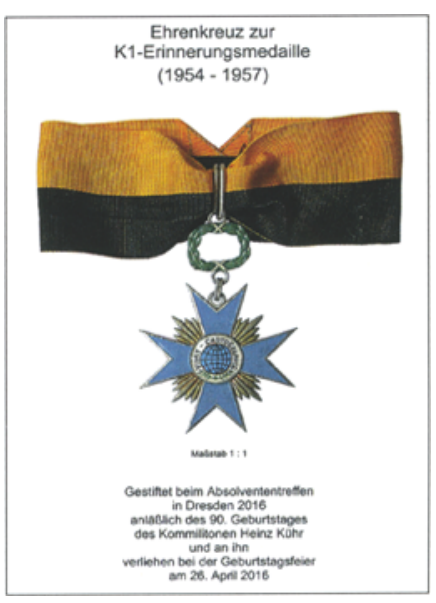

ten" am Blauen Wunder waren gekommen: Wolfgang Albrecht (Schwerin), Rolf Dörrstock (Bonn), Gisela Hachfeld (Bremen), Curt Hoffmann (Dresden), Cosima Klärner (Dresden), Rolf Opitz (Innsbruck), Dr. Werner Schirm (Berlin), Wolfgang Schmidt (Erkner), Kurt Ziesing (Erfurt) und die ehemalige Dozentin Helga Weber (Dresden).

Der Kommilitone Curt Hoffman, der bereits 1997 für die Absolventen eine Erinnerungsmedaille gestiftet und gefertigt hatte, schuf aus Anlass des Geburtstages für den Jubilar ein Ehrenkreuz zur Erinnerungsmedaille (siehe Abbildung) mit entsprechender Urkunde. Sichtlich gerührt nahm Heinz Kühr diese ganz persönliche Ehrerweisung seiner Kommilitonen entgegen. Im Verlauf seines beruflichen Wirkens war Heinz Kühr Stellvertretender Direktor der ehemaligen Ingenieurschule für Geodäsie und Kartographie in Dresden geworden.

Rolf Dörrstock, Bonn

DGfK-Mitglieder-

versammlung am

14. Juni 2016

in Potsdam

\section{Protokoll}

Die Mitgliederversammlung findet am 14. Juni 2016 von 16.15 Uhr bis 18.30 Uhr im Kongresshotel Potsdam statt. Es sind 53 Mitglieder anwesend.

\section{Tagesordnung:}

1. Begrüßung und Feststellung der ordnungsgemäßen Einladung und der Beschlussfähigkeit

2. Ehrung der verstorbenen Mitglieder

3. Genehmigung der Tagesordnung

4. Genehmigung des Protokolls der letzten Mitgliederversammlung

5. Ehrung verdienter Mitglieder 